\title{
Staircase in the Electron Mobility of a ZnO Quantum Dot Assembly due to Shell Filling
}

\author{
A. L. Roest, J. J. Kelly, and D. Vanmaekelbergh* \\ Debye Institute, Utrecht University, P.O. Box 80000, 3508 TA Utrecht, The Netherlands
}

E. A. Meulenkamp

Philips Research Laboratories, WB 62, Prof. Holstlaan 4, 5656 AA Eindhoven, The Netherlands

(Received 21 December 2001; published 27 June 2002)

\begin{abstract}
Electron transport in an assembly of $\mathrm{ZnO}$ quantum dots has been studied using an electrochemically gated transistor. The electron mobility shows a stepwise increase as a function of the electron occupation per quantum dot. When the occupation number is below two, transport occurs by tunneling between the $S$ orbitals. Transport becomes 3 times faster when the occupation number is between two and eight; tunneling now occurs between the $P$ orbitals. Electron transport is thus critically determined by the quantum properties of the building blocks.
\end{abstract}

DOI: 10.1103/PhysRevLett.89.036801

For many II-VI and III-V compounds synthetic routes have been developed which yield nearly monodisperse colloidal nanocrystals with a well-controlled size in the 1-10 $\mathrm{nm}$ range. These insulating nanocrystals show discrete atomiclike valence (hole) and conduction (electron) levels due to size confinement; the energy level spectrum can be tuned, within certain limits, by variation of the size of the dots. They are, therefore, called artificial atoms or quantum dots. Colloidal nanocrystalline quantum dots with their tunable energy levels [1,2] are promising building blocks for novel electrical and optoelectrical devices. A field-effect transistor (FET) with a single CdSe quantum dot has been studied [3]; this work forms an extension of more elaborate studies of electron transport through quantum dots [4-6] and quantum dot molecules [7-9]. In addition, electroluminescent [10,11] and electrochromic [12] devices based on assemblies of quantum dots have been described. The performance of such devices is critically dependent on the electronic properties of the active film. Despite this, the characteristics of electron transport in an assembly of quantum dots has not received much attention.

The electronic conductivity in an assembly of nanocrystalline quantum dots will be determined by the coupling between the atomiclike orbitals of neighboring nanocrystals. If the electronic coupling is weak, electron transport is not coherent. In this regime, electron transport can be thermally activated due to the effect of Coulomb blockade [13-15]. For an experimental study of possible quantum effects in the electronic conduction it is necessary to be able to vary the number of charge carriers per quantum dot in a controlled way. Furthermore, the effects of structural and electronic disorder must be small. This means that in the ideal case there should be no electronic states in the highest occupied-lowest unoccupied molecular orbital (HOMO-LUMO) gap and that all nanocrystals should have the same size and shape. We remark that the absence of electronic disorder and the precise control of the number of charge carriers per building block have proved to be a great asset for fundamental investigations of charge carrier
PACS numbers: 73.22.-f, 73.63.-b

transport [15] and superconductivity in crystals of organic molecules [16-19].

We have investigated storage and transport of electrons in an assembly of $\mathrm{ZnO}$ quantum dots. We used an electrochemically gated transistor which allows one to charge the quantum dot assembly uniformly, controlling the number of electrons per quantum dot. The increasing capacitance of the film during the injection of electrons reflects the sequential filling of the $S$ and $P$ atomiclike orbitals of the $\mathrm{ZnO}$ quantum dots. The electron mobility, derived from the source-drain conductance, shows a stepwise increase (staircase) as a function of the electron occupation per dot. The lowest discrete mobility is observed for an electron occupation number per dot smaller than two, thus corresponding to $S-S$ orbital tunneling. The second discrete mobility, which is about a factor of 3 higher, corresponds to $P-P$ orbital tunneling and is observed for an electron occupation between two and eight. The results suggest a third regime if there are more than eight electrons per quantum dot. We have thus shown that electron transport in a nanocrystalline assembly can be determined by the orbital set of the quantum dot building blocks.

Electrochemical injection of electrons into an assembly consisting of $\mathrm{ZnO}$ nanocrystals has been studied before [20]. There were, however, indications for storage of electrons in band gap states (traps), and for electrochemical reduction of the $\mathrm{ZnO}$ particles. One of us has developed a state-of-the art method for preparing homogeneous films consisting of nearly monodisperse $\mathrm{ZnO}$ quantum dots with diameters in the 3-6 nm range [21]. These assemblies could reversibly store up to 10 electrons per quantum dot without any sign of chemical degradation [22]. Encouraged by these results, we studied the electron transport properties of $\mathrm{ZnO}$ quantum dot films, consisting of nanocrystals, 3.9 and $4.3 \mathrm{~nm}$ in diameter, as a function of the electron occupation. We have used a transistor with an electrochemical gate. We will focus on the results obtained with $\mathrm{ZnO}$ films permeated with an aqueous electrolyte. 
First, we discuss the structure and operating principle of an electrochemically gated quantum dot transistor (Fig. 1). The electrochemical potentials, $\tilde{\mu}_{e}$, of the source and drain electrodes are controlled with respect to a $\mathrm{Ag} / \mathrm{AgCl}$ reference electrode by means of a bipotentiostat. A small potential difference $V_{s d}(1-10 \mathrm{mV})$ is maintained between source and drain to allow measurement of the linear conductance $R^{-1}=I_{s d} / V_{s d}$ of the $\mathrm{ZnO}$ film. The $\mathrm{ZnO}$ quantum dot layer equilibrates with the source (drain) electrode by adjustment of the electron density; the charge of the injected electrons is counterbalanced by incorporation of extra positive ions into the pores of the film (Fig. 1, inset). We remark that here the entire film is uniformly charged with electrons in contrast to the two-dimensional charging occurring in a conventional field effect transistor. In Fig. 2A the absorption spectrum of the $\mathrm{ZnO}$ film is compared with that of the nanocrystalline quantum dots in suspension. The slight redshift in the spectrum of the quantum dot layer relative to the suspension indicates a weak coupling of the quantum dots in the assembly.

The differential capacitance $C$ of the film [measured by the charge $\Delta Q\left(\tilde{\mu}_{e}\right)$ injected into the quantum dot layer per $50 \mathrm{meV}$ increase of the electrochemical potential $\left.\tilde{\mu}_{e}\right]$ reflects the quantum properties of the $\mathrm{ZnO}$ nanocrystalline building blocks. Results are shown in Fig. 3A for nanocrystals $3.9 \mathrm{~nm}$ in diameter. The onset of electron injection is at a potential $V \leq-0.65 \mathrm{~V}\left(\tilde{\mu}_{e} \geq 0.65 \mathrm{eV}\right)$; the capacitance function $C\left(\tilde{\mu}_{e}\right)$ shows two pronounced steps. Similar results are obtained with $4.3 \mathrm{~nm} \mathrm{ZnO}$ quantum dot layers where the onset occurs at a somewhat lower electrochemical potential. Integration of the differential

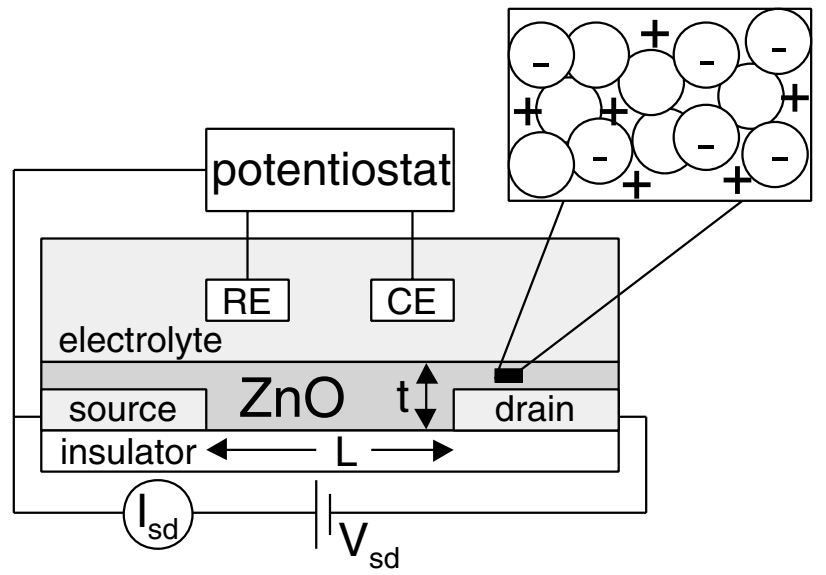

FIG. 1. Schematic view of the electrochemically gated transistor. The source-drain distance is denoted by $L(10 \mu \mathrm{m})$, and the thickness of the conductive channel is denoted by $t(\sim 200 \mathrm{~nm})$. The electrochemical potential of the active $\mathrm{ZnO}$ layer is controlled with respect to a $\mathrm{Ag} / \mathrm{AgCl}$ reference electrode (RE) using a bipotentiostat. The inset shows a detail of the active layer; the charge of the excess electrons in the quantum dots is counterbalanced by positive ions in the electrolyte. The conductance of the $\mathrm{ZnO}$ assembly is obtained by measurement of the source-drain current $I_{s d}: R^{-1}=I_{s d} / V_{s d}$. capacitance gives the cumulative charge in the quantum $\operatorname{dots} ; Q\left(\tilde{\mu}_{e}\right)=\int_{\tilde{\mu}_{e \text { onset }}}^{\tilde{\mu}_{e}} C\left(\tilde{\mu}_{e}\right) d\left(\tilde{\mu}_{e}\right)$. Since we know the number of nanocrystals in the film [23], we can calculate the average number of electrons per dot (i.e., occupation number $\langle n\rangle)$ as a function of the electrochemical potential. Figure 3B (blue curve) shows that $\langle n\rangle=1$ when $\tilde{\mu}_{e}$ is $0.83 \mathrm{eV}$, and that close to 8 electrons are present when $\tilde{\mu}_{e}$ is $1.2 \mathrm{eV}$.

The electrons in the $\mathrm{ZnO}$ quantum dots may occupy the delocalized atomiclike $S, P$, and higher conduction orbitals or be located in traps. In order to decide on the importance of traps, we measured the light absorbance by the $\mathrm{ZnO}$ layer under electron accumulation (Figs. 2B and $2 \mathrm{C}$; Fig. 3B, red curve). As soon as electrons are present in the film, an absorption decrease $(\Delta A)$ is found in a limited photon energy range between 3.4 and $3.8 \mathrm{eV}$, thus corresponding to the HOMO-LUMO transition. We found that the decrease of the absorbance (Fig. 3B, red curve) is proportional to the occupation number: $-\int \Delta A(h \nu) d(h \nu)=$ const $\times\langle n\rangle$; the fact that $\langle n\rangle$ goes to zero as $-\int \Delta A(h \nu) d(h \nu)$ goes to zero proves that electron storage in traps is not important. A similar conclusion can be drawn from the IR absorption at $h \nu \cong 250 \mathrm{meV}$, corresponding to the optical transition

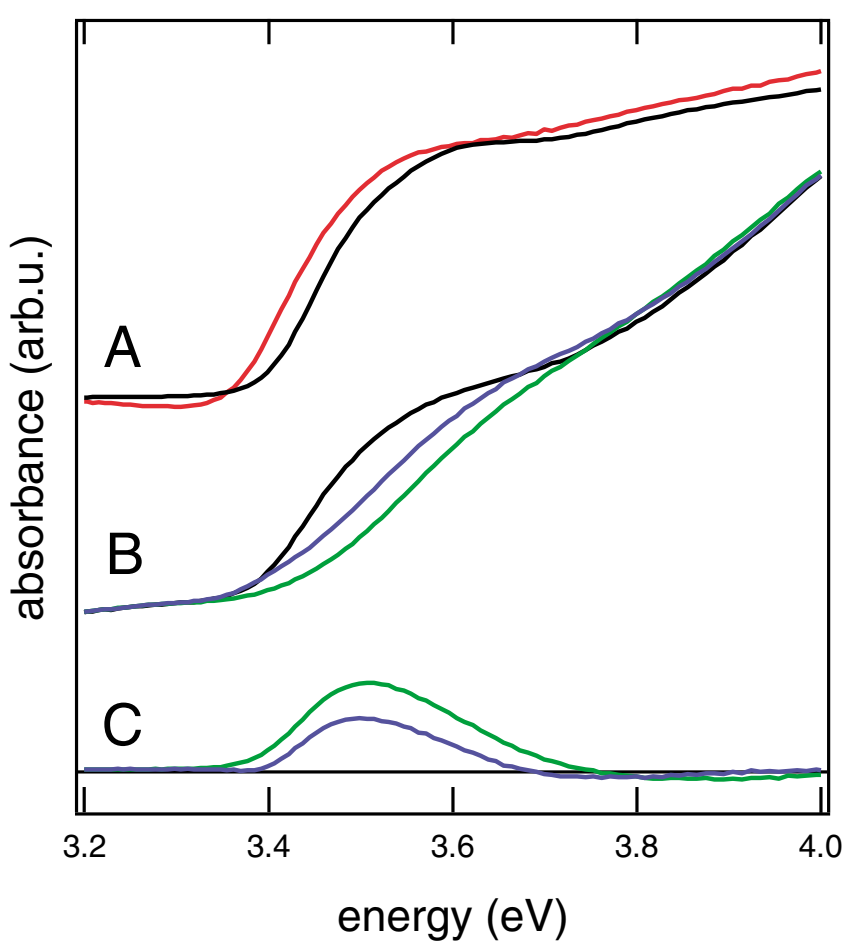

FIG. 2 (color). (A) The absorption spectra of the $\mathrm{ZnO}$ quantum dots (diameter $=3.9 \mathrm{~nm}$ ) in suspension (black line) and in the active layer of the transistor (red line). (B) Absorption spectra measured when electrons are electrochemically injected into the active layer of the transistor. Black line: $V=-0.6$ volt (no electrons present); blue line $V=-0.8$ volt, green line $V=-1.0$ volt . (C) The corresponding absorbance difference spectrum derived from (B). 


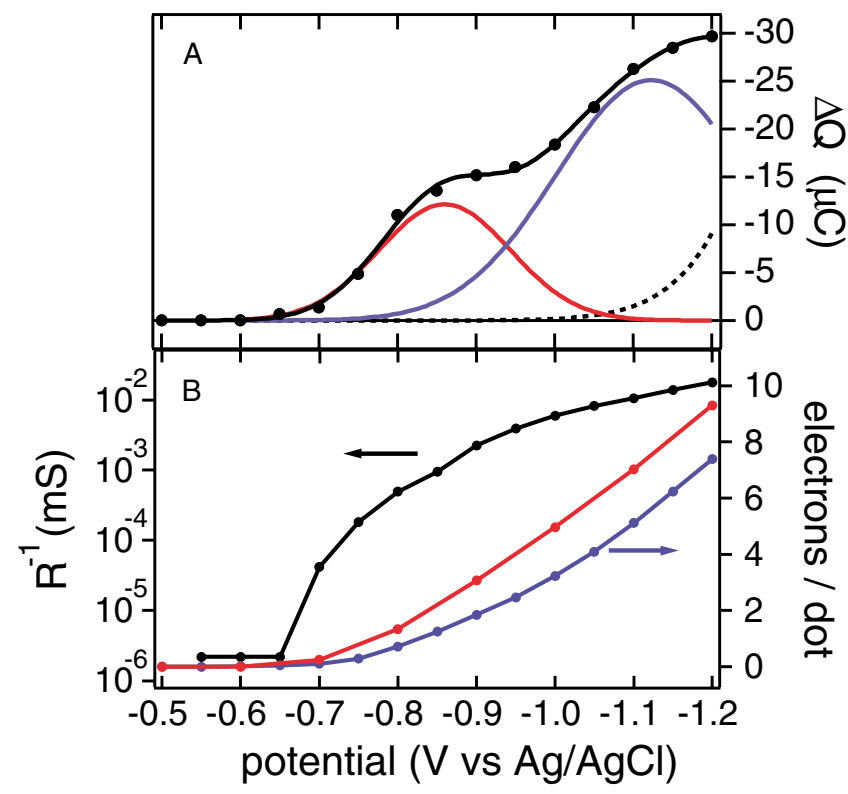

FIG. 3 (color). Electrochemical injection of electrons into a $\mathrm{ZnO}$ quantum dot film (diameter $=3.9 \mathrm{~nm}$ ). (A) The differential charge $\Delta Q\left(\tilde{\mu}_{e}\right)$, measured upon successive potential steps of $50 \mathrm{mV}$ as a function of the potential; the experimental results are represented by the dots $(\bullet)$. The fit of the differential charge function (black line) is explained in the text. (B) The average number of excess electrons per quantum dot in the active film (blue curve), the source-drain conductance $R^{-1}$ (black curve), and the integrated absorbance difference (red curve in arbitrary units, see Fig. 2C) as a function of the potential. The lines serve as a guide to the eye.

of an electron from an $S$ level to a $P$ level. The onset of IR absorption coincides with the onset of quenching of the HOMO-LUMO transition. We remark that trap-free storage of electrons in quantum dot films has also been observed with capped CdSe quantum dots [12]. The absence of electron trapping is further supported by the prompt and strong increase of the conductance of the quantum dot film as soon as electrons are present in the dots (Fig. 3B, black curve).

The discrete steps in the plot of the differential capacitance as a function of the potential (Fig. 3A) must reflect the atomiclike energy levels of the $\mathrm{ZnO}$ nanocrystals. The differential capacitance function can be fitted with a sum of single-electron Gaussian functions; each Gaussian function corresponds to the occupancy of one single-electron orbital. The width of the single-electron Gaussian function reflects the size distribution of the nanocrystals in the assembly. In Fig. 3A, the red curve is the sum of two Gaussian single-electron functions, accounting for the occupation of the $S$ orbitals. The blue curve accounts for the occupation of the $P$ orbitals (six electrons). From the spacing between the single-electron Gaussian functions in the fit (not shown), we found that the average electron-electron repulsion energy is $30 \pm 10 \mathrm{meV}$. This is considerably smaller than in an isolated nanocrystal (100-200 meV) [24]. This shows the effective screening of the electronic charge by the positive ions when the pores of the film are filled with an aqueous electrolyte solution. For the films consisting of $3.9 \mathrm{~nm}$ dots, the separation between the maxima of the $S$ - and $P$-level distributions is $250 \mathrm{meV}$, in agreement with the results of IR absorption spectroscopy. Because of the dispersion in particle size, we find an overlap between the $S$ - and $P$-level distributions; this has important consequences for electron transport in the film.

Figure $4 \mathrm{~A}$ presents the conductance $R^{-1}=I_{s d} / V_{s d}$ of the film (3.9 nm quantum dots) measured between source and drain as a function of the occupation number $\langle n\rangle$. The conductance increases linearly with $\langle n\rangle$ for $0 \leq\langle n\rangle \leq 1.5$, and for $2.5 \leq\langle n\rangle \leq 8$; note that in the second range the slope of the $R^{-1}-\langle n\rangle$ plot is steeper, showing that there are two regimes of electron transport characterized by a different electron mobility. We calculated the electron mobility from the measured conductance, using $\mu=\frac{L}{e Z t} \frac{\partial R^{-1}}{\partial n}[L$ is the channel length (source-drain distance), $Z$ is the width, and $t$ is the thickness of the conductive channel, $n$ is the electron density] and found $\mu=0.017 \mathrm{~cm}^{2} / \mathrm{V} \mathrm{s}$ for $0 \leq\langle n\rangle \leq 1.5$ and $\mu=0.066 \mathrm{~cm}^{2} / \mathrm{V}$ s for $2.5 \leq\langle n\rangle \leq 8$. Similar results are obtained with films of $4.3 \mathrm{~nm}$ nanocrystals (Fig. 4B); in this case $\mu$ shows three discrete steps. The fact that the observed mobilities are at least 3 orders of magnitude smaller than the critical mobility $\left(\frac{e \Delta^{2}}{h}=40 \mathrm{~cm}^{2} / \mathrm{V} \mathrm{s}, \Delta\right.$
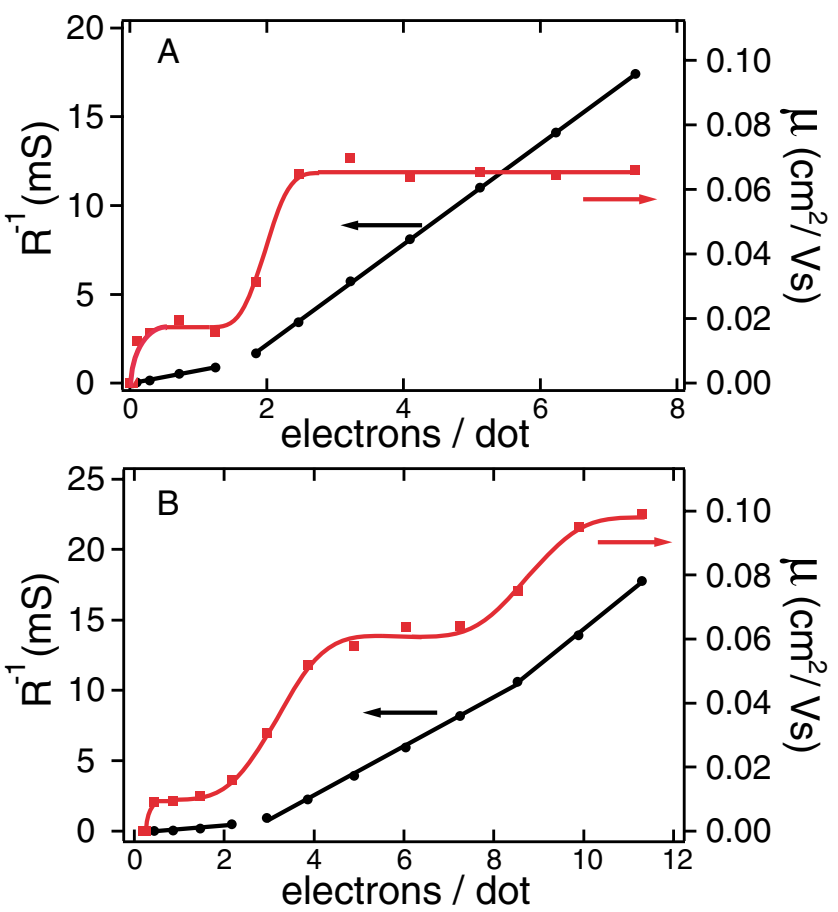

FIG. 4 (color). Transport properties of the $\mathrm{ZnO}$ films consisting of quantum dots 3.9 (A) and $4.3 \mathrm{~nm}$ (B) in diameter. We present the conductance $R^{-1}$ (black dots and line) and the corresponding electron mobility (red squares and line) as a function of the average number of electrons per quantum dot $\langle n\rangle$. The uncertainty in $\langle n\rangle$ and $R^{-1}$ for both experiments is about $10 \%$. 
being the diameter of the dots) shows that the electronic coupling between the quantum dots is weak and the transport is noncoherent [15]. This is in agreement with the observation that the optical properties of the quantum dot film are nearly identical to those of the quantum dot sol. We found that the mobilities are unaffected by the temperature in the accessible range between 5 and $35^{\circ} \mathrm{C}$ for films permeated with water. This observation is in accordance with the small Coulomb charging energy of about $k_{B} T$ at room temperature. Thus, electron transport occurs in the weak coupling regime, but is not attenuated by Coulomb blockade effects.

In the range $0 \leq\langle n\rangle \leq 2$, the electrons occupy predominantly the $S$ levels, while in the range $2 \leq\langle n\rangle \leq 8$ nearly all $S$ levels are occupied and the $P$ levels become progressively filled. We infer that the mobility in the range $0 \leq\langle n\rangle \leq 1.5$ corresponds to resonant tunneling between $S$ orbitals of adjacent quantum dots $\left(\mu_{S-S}=0.017 \mathrm{~cm}^{2} / \mathrm{V} \mathrm{s}\right)$, while the constant mobility in the range $2.5 \leq\langle n\rangle \leq 8$ reflects tunneling between $P$ orbitals $\left(\mu_{P-P}=0.066 \mathrm{~cm}^{2} / \mathrm{V} \mathrm{s}\right)$. Note that we do not observe a conducting-to-insulating transition at $\langle n\rangle=2$; this is due to the overlap of the distributions of the $S$ and $P$ energy levels. The significance of these tunnel mobilities can be appreciated by applying the Einstein-Smoluchowski formalism. Electron transport by $S$-to- $S$ and $P$-to- $P$ orbital tunneling corresponds to mobilities $\mu_{S-S}=\left(e / k_{B} T\right) \frac{\Delta^{2}}{\tau_{S-S}}$ and $\mu_{P-P}=\left(e / k_{B} T\right) \frac{\gamma \Delta^{2}}{\tau_{P-P}}$, respectively $(\Delta$ is the length over which the electron is transferred in one tunneling event, thus the diameter of the dot; $\tau^{-1}$ is the rate of tunneling between two orbitals of adjacent dots, and $\gamma$ accounts for the higher density of the $P$ orbitals per unit energy). The fact that $\mu_{P-P}$ is more than 3 times $\mu_{S-S}$ is in agreement with the above equations: $\gamma$ must be close to three. Moreover, because of better orbital overlap, $1 / \tau_{P-P}$ is expected to be somewhat larger than $1 / \tau_{S-S}$. In accordance, resonant tunneling via the $P$ orbitals of a single CdSe quantum dot is slightly faster than via the $S$ orbitals [25]. From $\mu_{S-S}=\left(e / k_{B} T\right) \frac{\Delta^{2}}{\tau_{S-S}}=0.017 \mathrm{~cm}^{2} / \mathrm{V} \mathrm{s}$ it follows that the tunnel rate between $S$ orbitals of adjacent $\mathrm{ZnO}$ dots $1 / \tau_{S-S}$ is $3 \times 10^{9} / \mathrm{s}$. By comparison, the rates of resonant tunneling in a single-dot device with metal/dot tunnel barriers of $1 \mathrm{~nm}$ in width are 2 orders of magnitude smaller [25].

The role of structural and electronic disorder in quantum dot solids has been anticipated theoretically [13]. In the $\mathrm{ZnO}$ quantum dot films studied here, the disorder is related to the size distribution of the dots and their random assembly (i.e., not close packed) in the film. These factors of inherent disorder may lead to variations in the degree of orbital overlap between the dots and the Coulomb charging energy. Experimental studies on the effects of disorder on the optical and electronic properties of quantum dot solids will be an important scientific issue in the coming years.

In summary, we have shown that the electronic properties of an assembly of $\mathrm{ZnO}$ nanocrystals are determined by the quantum nature of the building blocks. Solids that offer different quantum regimes of electrical transport are very promising for novel electrical and optoelectrical devices.

*Electronic address: D.A.M.Vanmaekelbergh@phys.uu.nl [1] H. Weller, Adv. Mater. 5, 88 (1993).

[2] A. P. Alivisatios, J. Phys. Chem. 100, 13226 (1996).

[3] D. L. Klein et al., Nature (London) 389, 669 (1997).

[4] R. C. Ashoori et al., Phys. Rev. Lett. 68, 3088 (1992).

[5] S. Tarucha et al., Phys. Rev. Lett. 77, 3613 (1996).

[6] M. Ciorga et al., Phys. Rev. B 61, R16 315 (2000).

[7] F. R. Waugh et al., Phys. Rev. Lett. 75, 705 (1995).

[8] T. H. Oosterkamp et al., Nature (London) 395, 873 (1998).

[9] R. H. Blick et al., Phys. Rev. Lett. 80, 4032 (1998).

[10] V. L. Colvin, M. C. Schlamp, and A. P. Alivisatos, Nature (London) 370, 354 (1994).

[11] B. O. Dabbousi, M. G. Bawendi, O. Onitsuka, and M. F. Rubner, Appl. Phys. Lett. 66, 1316 (1995).

[12] C. Wang, M. Shim, and P. Guyot-Sionnest, Science 291, 2390 (2001).

[13] F. Remacle and R.D. Levine, ChemPhysChem 2, 20 (2001).

[14] C. W. J. Beenakker, Phys. Rev. B 44, 1646 (1991).

[15] W. A. Schoonveld et al., Nature (London) 404, 977 (2000).

[16] J.H. Schön, C. Kloc, and B. Batlogg, Nature (London) 406, 702 (2000).

[17] J. H. Schön, C. Kloc, and B. Batlogg, Nature (London) 408, 549 (2000).

[18] J.H. Schön, A. Dodabalapur, C. Kloc, and B. Batlogg, Science 290, 963 (2000).

[19] J.H. Schön, C. Kloc, E. Bucher, and B. Batlogg, Nature (London) 403, 408 (2000).

[20] P. Hoyer and H. Weller, J. Phys. Chem. 99, 14096 (1995).

[21] E. A. Meulenkamp, J. Phys. Chem. B 102, 5566 (1998).

[22] E. A. Meulenkamp, J. Phys. Chem. B 103, 7831 (1999).

[23] The size of the $\mathrm{ZnO}$ nanocrystals was determined with x-ray diffraction and transmission electron microscopy (TEM). The degree of polydispersity was obtained from TEM size histograms; $85 \%-90 \%$ of the nanocrystals have a size within $20 \%$ of the mean diameter. Optically transparent films were deposited by spin coating the suspension on ITO/glass or gold/glass substrates, followed by drying at $90{ }^{\circ} \mathrm{C}$ in air for $15 \mathrm{~min}$. A previous scanning electron microscopy study has shown that the films are homogeneous [22]. The number of quantum dots in the layers was calculated from the amount of zinc determined with the elemental analysis technique ICP-OES and the size of the nanocrystals.

[24] A. Franceschetti, A. Williamson, and A. Zunger, J. Phys. Chem. B 104, 3398 (2000).

[25] E. P. A. M. Bakkers et al., Nano Lett. 1, 551 (2001). 